\title{
Constitutive model for plasticity in an amorphous polycarbonate
}

\author{
A. Fortunelli \\ Molecular Modeling Laboratory, Istituto per i Processi Chimico-Fisici del C.N.R., v. G. Moruzzi, 1-56124, Pisa, Italy \\ M. Ortiz \\ Division of Engineering and Applied Science, California Institute of Technology, Pasadena, California 91125, USA
}

(Received 29 May 2007; published 19 October 2007)

\begin{abstract}
A constitutive model for describing the mechanical response of an amorphous glassy polycarbonate is proposed. The model is based on an isotropic elastic phase surrounded by an $\mathrm{SO}(3)$ continuum of plastic phases onto which the elastic phase can collapse under strain. An approximate relaxed energy is developed for this model on the basis of physical considerations and extensive numerical testing, and it is shown that it corresponds to an ideal elastic-plastic behavior. Kinetic effects are introduced as rate-independent viscoplasticity, and a comparison with experimental data is presented, showing that the proposed model is able to capture the main features of the plastic behavior of amophous glassy polycarbonate.
\end{abstract}

DOI: 10.1103/PhysRevE.76.041806 PACS number(s): 61.41.+e, 46.35.+z, 46.15. $-\mathrm{x}, 83.10 .-\mathrm{y}$

\section{INTRODUCTION}

The inelastic deformation of glassy polymers in the solid state is of considerable technological importance, e.g., as the basis of a number of industrial processes such as drawing and lamination, and as such has long been the subject of research (cf., e.g., [1]). Prominent among engineering polymers is bisphenol-A-polycarbonate (BPA-PC), often simply called polycarbonate, a linear chain thermoplastic whose chemical structure is shown in Fig. 1. For instance, polycarbonate is the nonmodified bulk polymer with the highest impact resistance in the 70-400 K temperature range, 15-20 times larger than PMMA. Polycarbonate is generally amorphous and transparent, and remains ductile at temperatures well below its glass transition $T_{g}=418 \mathrm{~K}$. These properties render polycarbonate the technopolymer of choice for applications requiring good thermal resistance, transparency, and shock resistance over a wide range of temperature, including canopies for supersonic aircraft, helmets, windshields, bulletresistant laminates, food-processing equipment, computer housing, eye goggles, and others.

Despite this technological importance, the physics of glassy-polymer plasticity remains the subject of considerable conjecture. Indeed, a brief survey of the existing models serves to highlight the present lack of consensus regarding fundamental mechanisms of plastic deformation and their effective mathematical modeling. Argon [2] suggested that plastic shear deformation in glassy polymers is the result of localized shear transformations occurring in small atom clusters. In this scenario, the rate of plastic deformation is nucleation controlled. Argon described the shear transformations as the formation of pairs of molecular kinks extending over the molecular diameter of the polymer chains [2] or as eigendeformations deforming the volume elements into oblate spheroids [3]. Further extensions of this model [4-6] postulate two distinct barriers to deformation: an isotropic, viscoplastic barrier to chain segment rotations induced by local shear transformations and an isotropic barrier to chain alignment. Each barrier is assumed to evolve independently with strain. The resulting constitutive model is able to capture the main features of the stress-strain curves of polycarbonate.
Argon [3] also argued that nonuniformity of local structure, especially in the form of low-density regions containing an excess of free volume, plays an important role in shaping plastic behavior. According to this model, these weak regions are more abundant and percolate in the melt, thus giving rise to its compliant behavior $[7,8]$, whereas the weak regions are spatially isolated in the polymer below the glass transition temperature, where they act as preferential sites for plastic deformation $[9,10]$. Perez and co-workers [11-13] have modeled the correlated dynamics of weak low-density regions, which they call quasi-point-defects. According to this model, the relaxation times of the quasi-point-defects are widely distributed, ranging from a low value corresponding to the activation of an elementary molecular motion to a high value representing the time required for two sheared microdomains to coalesce, thus giving rise to a complex collective and hierarchically correlated dynamics. Stachurski [14] instead posited that deformation and yield in amorphous polymers can be understood in terms of the integrity of the network of the strongest intermolecular bonds, or constriction points, in a weaker, lower-density matrix. The important role of heterogeneity and of the varying degrees of crystallization over the polymer, as well as the dynamics and percolation of the lose and cohesive regions, has been recognized by a number of authors [15-18]. The mean size of these heterogeneities has been experimentally estimated to be of the order of $4-5 \mathrm{~nm}$.

The picture of a noncontinuous nanometric structure of the glassy matrix and its bearing with the plastic process has been confirmed and refined by recent atomistic simulations of the plastic behavior of polycarbonate (cf. [19] and discussion of previous computational modeling therein). These simulations have provided detailed insight into the funda-

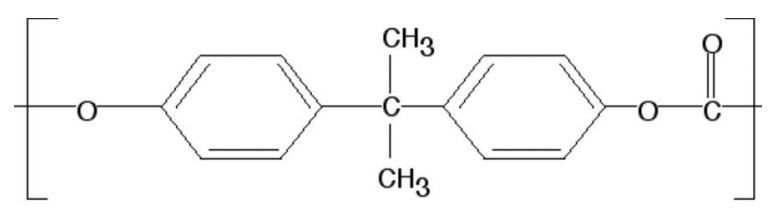

FIG. 1. Schematic structure of bis-phenol-A polycarbonate. 


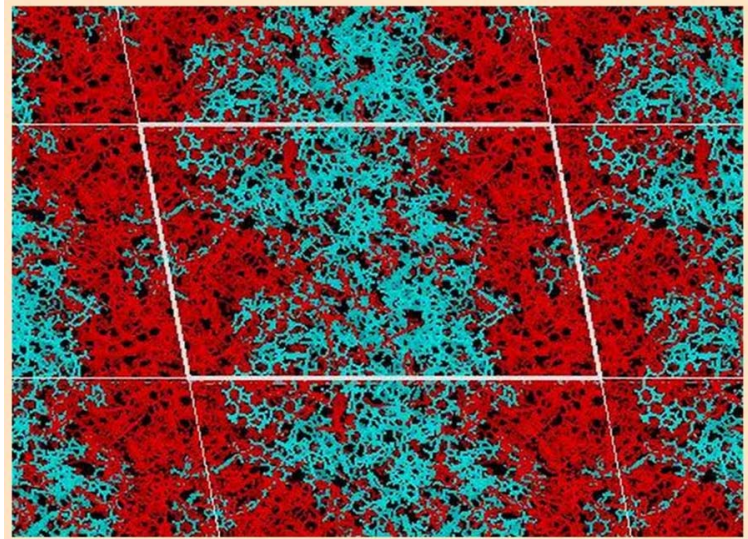

FIG. 2. (Color online) A view of the atoms involved in a typical plastic transition of BPA-PC [light (blue)], as opposed to atoms which do not undergo significant motions [dark (red)]. Reused with permission from Fortunelli, Geloni, and Lazzeri [19].

mental mechanisms of plastic deformation in glassy polymers and effectively removed much of the speculation surrounding their precise nature. The simulations show that plastic yielding in polycarbonate presents a collective and cooperative character: individual segmental motions percolate in an avalanche motion involving a sizable fraction of the cell. Such a motion, however, is not random, but rather takes the form of a nanoscopic lamination. Thus, with increasing stress the system separates into two alternating phases in the manner of solid-solid phase transition [20]: (i) a region substantially unmodified with respect to the ideal structure that is prevalent in the initial elastic regime and (ii) a region that is transformed into a different ideal structure that becomes increasing prevalent in the plastic regime. The volume and one of the principal stretches of the transformed region remain invariant throughout deformation, which shows that the transformed phase deforms in shear. The interfaces between the two faces are somewhat irregular, as expected for such amorphous materials, but are clearly recognizable as planar interfaces. A typical plastic transition in polycarbonate reproduced from the atomistic simulations of Ref. [19] is shown in Fig. 2. The thickness of the observed lamellae is very small: roughly $\sim 5 \mathrm{~nm}$, in agreement with experimental estimates $[16,18]$. Their extension, or persistence length, could not be determined from the molecular dynamics (MD) simulations due to the limited size of the cell.

In this paper we take the observation of lamellar structures reported in [19] as a basis for formulating a continuum model of glassy-polymer plasticity. The fundamental picture that arises from atomistic calculations is that glassy-polymer plasticity is a process of accommodation that exploits the existence of two types of low-energy phases: the undeformed structure of the polymer and deformed structures that differ from the former by a transformation shear. Conveniently, the transformation shear of polycarbonate can be deduced from the atomistic simulations reported in [19]. Since the parent phase is essentially amorphous, the plane and direction of the transformation shear are arbitrary, which introduces an infin- ity of wells, parametrized by $\mathrm{SO}(3)$, in the phase diagram. This multiwell phase diagram enables the material to reduce its energy while preserving its average deformation by developing microstructure. These microstructures are fine mixtures of phases that are in equilibrium and satisfy compatibility conditions at all interfaces. The macroscopic effect of the formation of microstructure is to introduce a yield point and a compliant plastic branch in the stress-strain curve. As the macroscopic deformation is increased, the volume fractions of the phases evolves by a process of interfacial motion. That process is dissipative and renders the macroscopic behavior hysteretic.

The central problem addressed in this paper is that of ascertaining the macroscopic behavior of the material from its nanoscopic energetics and kinetics, as described by the multiwell energy and interfacial mobility just outlined, leading to a continuum model that can be used in large-scale engineering simulations. Conveniently, a number of mathematical tools are presently in existence that can be resorted to in order to forge that connection. In the calculus of variations, the process of accommodation described in the foregoing is known as relaxation (cf., e.g., [21,22]). Relaxation replaces an energy functional that gives rise to microstructure by a relaxed energy functional that represents exactly the macroscopic behavior of the material. The computation of the relaxed energy functional requires the evaluation of all possible microstructures that can arise in response to a macroscopic affine deformation. Often, such exhaustive evaluation is not possible, and a fallback position is to consider special classes of microstructures that are amenable to analysis, leading to a partially relaxed energy functional. Motivated by the atomistic calculations reported in [19], in this work we specifically consider a class of microstructures known as sequential laminates [21,23], which are obtained recursively by fitting laminates within laminates. This process of lamination results in the so-called rank-1 convex hull of the energy density, which in general lies above the completely relaxed energy density. We account for interfacial dissipation by means of a simple model proposed by Aubry, Fago, and Ortiz [24]. The proposed approach provides a physics-based and analytically tractable means of bridging the atomistic and continuum scales of glassy polymers. The resulting model provides a simple constitutive framework for describing the plasticity of glassy polymers.

\section{UNRELAXED ENERGY}

Given $\mathbf{F}$, the deformation tensor, written according to the polar decomposition as $\mathbf{F}=\mathbf{R} \mathbf{U}$ with $\mathbf{R}$ a pure rotation and $\mathbf{U}$ the (symmetric positive) stretch tensor, one can always find [25] a reference system (the principal stretch system) such that $\mathbf{U}=\operatorname{diag}\left\{\lambda_{1}, \lambda_{2}, \lambda_{3}\right\}$, with $\lambda_{i} \geqslant 0$. Let us assume for convenience that the $\left\{\lambda_{i}\right\}$ are ordered: $\lambda_{3} \geqslant \lambda_{2} \geqslant \lambda_{1} \geqslant 0$.

We assume that the material is isotropic. By material frame indifference, the free energy can depend only on the stretch tensor $\mathbf{U}$. For isotropic materials, the expression can depend only on its eigenvalues $\left\{\lambda_{i}\right\}$ or, equivalently, on the invariants of $\mathbf{U}$. Given the isochoric character of the plastic phenomenon (which implies incompressibility-i.e., that the 
third invariant is constant along the transformation: $I_{3}$ $\left.=\lambda_{1} \lambda_{2} \lambda_{3}=1\right)$ and the theory of rubber elasticity, we will assume that in the elastic regime the free energy depends only on the first invariant $I_{1}$, thus obtaining the following (neoHookean) form:

$$
W^{0}(\mathbf{F})=\frac{\mu}{2}\left(\lambda_{1}^{2}+\lambda_{2}^{2}+\lambda_{3}^{2}-3\right)=\frac{\mu}{2}\left(I_{1}-3\right),
$$

where $\mu$ is the shear modulus. Based on the results of the atomistic simulations of Ref. [19], according to which the elementary steps of the plastic transition correspond to laminates, we further assume that the elastic structure is surrounded by plastic structures which are connected to it via pure shear transformations-i.e., transformations characterized by the eigenvalues $\lambda_{2}=1$ and $\lambda_{1}=1 / \lambda_{3}$ (in Appendix A we show that this formalism is equivalent to the usual definition of shear deformation matrices). By requiring isotropy and invoking self-similarity, we finally assume that we can extrapolate the nanoscopic behavior at an appropriate, larger (mesoscopic) length scale. The unrelaxed energy expression must therefore correspond to an isotropic double well. In other words, starting from $W^{0}$ and moving along any direction in pure shear, one runs into $W^{1}$ which lies at higher energy by a term $\Delta U$ and attains its minimum for these values of $\lambda_{i}:\left\{\lambda_{2}=1 ; \lambda_{3}=\eta ; \lambda_{1}=1 / \eta\right\}$-i.e.,

$$
W^{1}(\mathbf{F})=\Delta U+\frac{\mu}{2}\left[\eta^{2} \lambda_{1}^{2}+\lambda_{2}^{2}+\left(\lambda_{3} / \eta\right)^{2}-3\right] .
$$

In keeping with the atomistic simulations of Ref. [19] (see Figs. 4 and 5 therein), $W^{1}$ represents the energy of a structure which lies at higher energy at zero strain, but crosses with $W^{0}$ and becomes the lowest-energy one at large shear deformations, so that $W^{0}$ dynamically collapses onto it (plastic collapse). Since we suppose to run into one such structure along any direction in pure shear, what we really have is an $\mathrm{SO}(3)$ continuum of neighboring states. It can be noted that, for simplicity, we have supposed constancy of the elastic shear modulus-i.e., constancy of $\mu$. The value of $\eta$ is related to the amount of plastic yield, or irreversible plastic deformation, and can be inferred from experimental data: for BPA-PC at room temperature (RT) $\eta \approx 1.5-2.0 . \Delta U$ represents the energy stored into the material by virtue of the plastic transformation: see Ref. [19] for numerical values of $\Delta U$ for BPA-PC. Due to the assumption of isotropy, $\Delta U$ has the same value for all the plastic structures.

By taking $\mu / 2$ as the energy unit and correspondingly defining a normalized value of $\Delta U$ as: $\delta U=2 \Delta U / \mu$, the final expression for the unrelaxed energy is

$$
\begin{aligned}
W_{\text {unrelax }}(\mathbf{F})= & \min \left\{W^{0}(\mathbf{F}) ; W^{1}(\mathbf{F})\right\} \\
= & \min \left\{\lambda_{1}^{2}+\lambda_{2}^{2}+\lambda_{3}^{2}-3 ; \delta U+\eta^{2} \lambda_{1}^{2}+\lambda_{2}^{2}\right. \\
& \left.+\left(\lambda_{3} / \eta\right)^{2}-3\right\} .
\end{aligned}
$$

In Appendix B it is shown that Eq. (3) coincides with that obtainable through the usual definition of shear deformation matrices. For ease of notation, it is convenient to define $\gamma$ as an alternative parameter characterizing the amount of plastic yield: $\gamma=\eta-1 / \eta$.

\section{RELAXED ENERGY}

The constitutive equation (3) is in a form similar to those considered in nonlinear elastostatics [25]. It is therefore amenable to analysis via the mathematical machinery developed for describing phase transitions in crystalline systems $[20,22]$. Essentially, the problem reduces to finding the quasiconvex envelope $W^{\mathrm{qc}}(\mathbf{F})$ of Eq. (3)-i.e., the largest function $f$ lying below $W_{\text {unrelax }}(\mathbf{F})$ and such that

$$
\int_{\Omega} f(\mathbf{A}+\boldsymbol{\nabla} \cdot \mathbf{v}(\mathbf{x})) d \mathbf{x} \geqslant \int_{\Omega} f(\mathbf{A}) d \mathbf{x}
$$

where $\mathbf{v}(\mathbf{x})$ is a smooth perturbation of the affine deformation Ax, vanishing on the boundary of $\Omega$, but otherwise arbitrary. To this aim, special but very useful constructions are "laminates"-i.e., piecewise affine deformation functions given by the alternance of two (in the case of first-order laminates) or more (in the case of higher-order laminates) deformation tensors differing by rank-1 matrices, the latter requirement being a consequence of Hadamard compatibility conditions; see Ref. [22] for more details. The largest such function lying below $W_{\text {unrelax }}(\mathbf{F})$ is called the lamination convex envelope $W^{\mathrm{lc}}(\mathbf{F})$. Proof of quasiconvexity has a particular significance, as it is known [26] that it is equivalent to weak sequential lower semicontinuity of the associated freeenergy integral and thus to the convergence of minimizing sequences in the case that an absolute minimum is not attained due to frustration of spatially uniform states (an experimentally observed microstructure can be thought of as an element of a minimizing sequence that oscillates more and more finely).

The model here considered differs with respect to previously known cases. For example, in martensitic materials one has only a finite number-say, 3 or 6-of energy wells $[27,28]$. Nematic elastomers [29] have an energy expression similar to Eq. (3) —indeed, the recent analytic solution given by DeSimone and Dolzmann [30-32] has inspired the present work-as the wells outside the "origin" (the reference structure of the high-temperature phase) are arranged in an $\mathrm{SO}$ (3) continuum of directions (isotropy). However, in nematic elastomers below the transition temperature the origin lies above the quasiconvex envelope of the wells off the origin, so that it does not interfere with its construction. In the present case, instead, one has the necessity to combine both the $W^{0}$ well with the $\left\{W^{1}\right\}$ wells and the various $\left\{W^{1}\right\}$ wells among themselves to quasiconvexify the energy. In other words, we have an asymmetric two-well isotropic model with pure shear. We call the model "asymmetric," because the energy difference between the bottoms of the wells, $\delta U$, introduces an additional degree of freedom which cannot be got rid of through a simple shifting or rescaling of the energy.

Building up on the results of DeSimone and Dolzmann [30-32] and of Šilhavý [33], we will take advantage of the fact that $W^{1}(\mathbf{F})$-after subtracting the constant energy shift $\delta U$-is of the form considered in Ref. [31] when we take $p=2, \gamma_{1}=1 / \eta, \gamma_{2}=1$, and $\gamma_{3}=\eta$. From their work we thus 


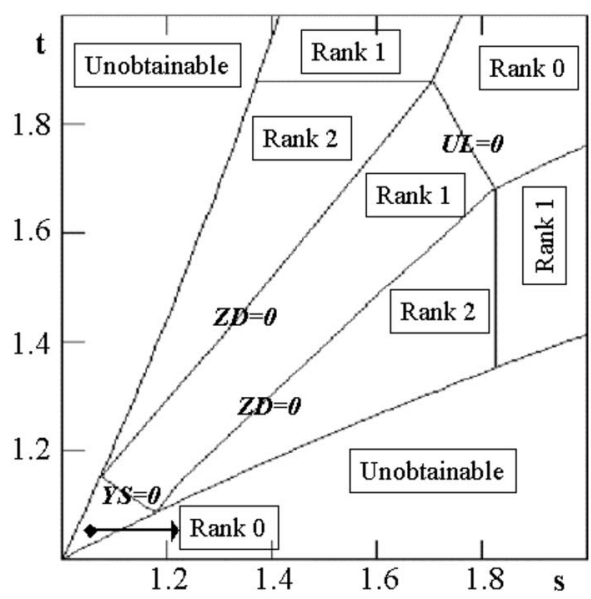

FIG. 3. Phase diagram of the proposed construction, Eq. (16), for the lamination convex envelope of the unrelaxed energy expression, Eq. (3). The curves $Y S=0, U L=0$, and $Z D=0$ delimiting the various lamination regions are defined in the text. The rank order of the laminations is shown in the appropriate regions. The values of the parameters are $\eta=1.55$ and $\delta U=0.431$.

know the lamination convex envelope of the $W^{1}(\mathbf{F})$ term (which in their case they prove to coincide with the quasiconvex envelope). The corresponding phase diagram can be divided into (a) a "liquid" phase $\left\{\lambda_{1} \geqslant 1 / \eta ; \lambda_{3} \leqslant \eta\right\}$, in which the relaxed energy $W_{\text {relax }}^{1}(\mathbf{F})$ coincides with $\delta U$ and the optimal microstructures are double laminates; (b) two "smectic" regions $\left\{\lambda_{3} \geqslant \eta ; \lambda_{1} \geqslant 1 / \sqrt{\eta \lambda_{3}}\right\}$ and $\left\{\lambda_{1} \leqslant 1 / \eta ; \lambda_{3} \leqslant \sqrt{\eta / \lambda_{1}}\right\}$, in which $W_{\text {relax }}^{1}(\mathbf{F})$ only depends on $\lambda_{3}$ or $\lambda_{1}$, respectively, and the optimal microstructure is a single laminate; (c) a "solid" region (encompassing the rest of the phase diagram), in which no microstructures are formed and the relaxed energy coincides with the unrelaxed one.

\section{A. Construction of a lamination convex envelope}

We make the assumption that the material is incompressible, in analogy with Ref. [31], so that $\lambda_{1} \lambda_{2} \lambda_{3}=1$. For elastic materials, this assumption implies that the material is neoHookean: this is accurate for rubbers, somewhat less accurate for the elastic response of amorphous polymers in the glassy state. However, here we are studying the plastic phenomenon which is essentially isochoric (as confirmed by the atomistic simulations of Ref. [19]), so that we need to consider only constant-volume laminations in the construction of the lamination convex envelope. In order to draw the phase diagram, we use the same variables introduced by DeSimone and Dolzmann [31]: $s=\lambda_{3}$ and $t=1 / \lambda_{1}$, where $\lambda_{3}$ is the largest singular value of $\mathbf{F}$ and $\lambda_{1}$ is its smallest (we recall that the eigenvalues are ordered). $\lambda_{2}$ is then recovered by the volume constraint as $\lambda_{2}=t / s$. Given the ordering of the eigenvalues $\left(\lambda_{3} \geqslant \lambda_{2} \geqslant \lambda_{1} \geqslant 0\right)$, the only accessible region of the phase diagram is that defined by the two constraints $t$ $\geqslant \sqrt{s}$ and $t \leqslant s^{2}$; see Fig. 3. In this section, we propose an approximate construction based on laminations, and we give numerical evidence that it corresponds to the lamination convex envelope of the original unrelaxed energy expression,
Eq. (3). We anticipate that we are not able to prove that it also corresponds to the quasiconvex envelope of Eq. (3), but as the lamination convex envelope of Eq. (3), we suggest that in any case it represents the only experimentally accessible response of the system to strain.

\section{Neighborhood of the $\lambda_{2}=1$ line}

We will concentrate for the sake of simplicity only on the lower half of the phase diagram: $\lambda_{2} \leqslant 1$ or $t \leqslant s$. Let us start by considering a deformation tensor $\mathbf{F}$ lying on the $\lambda_{2}=1$ line. Here, the optimal lamination is naturally suggested by analogy with the two-dimensional case. Given a generic deformation tensor $\mathbf{F}$ with eigenvalues $\left\{\lambda_{2}=1 ; \lambda_{3}, \lambda_{1}\right\}$, we can always bring it to a standard (upper-diagonal unit) form (see Appendix B):

$$
\mathbf{F}=\left(\begin{array}{lll}
1 & 0 & \alpha \\
0 & 1 & 0 \\
0 & 0 & 1
\end{array}\right),
$$

where $\left\{\lambda_{3}, \lambda_{1}=\sqrt{1+\alpha^{2} / 4} \pm \alpha / 2\right\}$ and $\alpha=\lambda_{3}-\lambda_{1}$.

By fixing $\lambda_{2}=1$ (as suggested by the analogy with the two-dimensional case), we now consider a lamination between the elastic $W^{0}(\mathbf{F})$ well and one of the plastic $W^{1}(\mathbf{F})$ wells:

$$
\begin{gathered}
\mathbf{F}=\nu \mathbf{F}_{1}+(1-\nu) \mathbf{F}_{2}=\nu\left(\begin{array}{ccc}
1 & 0 & \alpha_{1} \\
0 & 1 & 0 \\
0 & 0 & 1
\end{array}\right)+(1-\nu)\left(\begin{array}{ccc}
1 & 0 & \alpha_{2} \\
0 & 1 & 0 \\
0 & 0 & 1
\end{array}\right), \\
W_{\text {relax }}(\mathbf{F})=\nu W^{0}\left(\mathbf{F}_{1}\right)+(1-\nu) W^{1}\left(\mathbf{F}_{2}\right)
\end{gathered}
$$

where $\nu$ is the fraction of the $\mathbf{F}_{1}$ phase. The condition $\nu \alpha_{1}$ $+(1-\nu) \alpha_{2}=\alpha$ gives

$$
\nu=\frac{\alpha_{2}-\alpha}{\alpha_{2}-\alpha_{1}}, \quad 1-\nu=\frac{\alpha-\alpha_{1}}{\alpha_{2}-\alpha_{1}} .
$$

The optimal lamination can be obtained by minimizing $W_{\text {relax }}(\mathbf{F})$ with respect to $\alpha_{1}$ and $\alpha_{2}$. By imposing the zeroing of the derivatives of $W_{\text {relax }}(\mathbf{F})$ with respect to $\alpha_{1}$ and $\alpha_{2}$, one obtains a system of two equations in two unknowns, which can be easily solved numerically to give the standard Gibbs (isotangent or common tangent) construction. The solution is that lamination is effective when $\alpha$ belongs to the interval

$$
\alpha_{1}^{\min } \leqslant \alpha \leqslant \alpha_{2}^{\max }
$$

where $\alpha_{1}^{\min }$ and $\alpha_{2}^{\max }$ denote the optimal values, corresponding to the lower and upper extremes of this interval, respectively [34].

Let us now consider the $\lambda_{2} \neq 1$ case. Given a generic deformation tensor $\mathbf{F}$ with eigenvalues $\left\{\lambda_{2} \neq 1 ; \lambda_{3}, \lambda_{1}\right\}$, we cannot bring it to an upper-diagonal unit form, but to a similar standard form (see Appendix B): 


$$
\mathbf{F}=\left(\begin{array}{ccc}
\frac{1}{\sqrt{\lambda_{2}}} & 0 & \alpha \\
0 & \lambda_{2} & 0 \\
0 & 0 & \frac{1}{\sqrt{\lambda_{2}}}
\end{array}\right)
$$

where $\left\{\lambda_{3}, \lambda_{1}=\sqrt{1 / \lambda_{2}+\alpha^{2} / 4} \pm \alpha / 2\right\}$ and $\alpha=\lambda_{3}-\lambda_{1}$, as before. By holding the value of $\lambda_{2}$ fixed, we now consider a lamination between the elastic $W^{0}(\mathbf{F})$ well and one of the plastic $W^{1}(\mathbf{F})$ wells:

$$
\mathbf{F}=\nu \mathbf{F}_{1}+(1-\nu) \mathbf{F}_{2}=\nu\left(\begin{array}{ccc}
\frac{1}{\sqrt{\lambda_{2}}} & 0 & \alpha_{1} \\
0 & \lambda_{2} & 0 \\
0 & 0 & \frac{1}{\sqrt{\lambda_{2}}}
\end{array}\right)+(1-\nu)\left(\begin{array}{ccc}
\frac{1}{\sqrt{\lambda_{2}}} & 0 & \alpha_{2} \\
0 & \lambda_{2} & 0 \\
0 & 0 & \frac{1}{\sqrt{\lambda_{2}}}
\end{array}\right),
$$

$$
W_{\text {relax }}(\mathbf{F})=\nu W^{0}\left(\mathbf{F}_{1}\right)+(1-\nu) W^{1}\left(\mathbf{F}_{2}\right)
$$

where

$$
\begin{gathered}
W^{0}\left(\mathbf{F}_{1}\right)=\lambda_{2}^{2}+\frac{2}{\lambda_{2}}+\alpha_{1}^{2}-3 \\
W^{1}\left(\mathbf{F}_{2}\right)=\lambda_{2}^{2}+\delta U+\eta^{2}\left(\frac{1}{\lambda_{2}}+\frac{\alpha_{2}^{2}}{2}-\alpha_{2} \sqrt{\frac{1}{\lambda_{2}}+\alpha_{2}^{2} / 4}\right) \\
+\frac{1}{\eta^{2}}\left(\frac{1}{\lambda_{2}}+\frac{\alpha_{2}^{2}}{2}+\alpha_{2} \sqrt{\frac{1}{\lambda_{2}}+\alpha_{2}^{2} / 4}\right)-3 .
\end{gathered}
$$

The condition $\nu \alpha_{1}+(1-\nu) \alpha_{2}=\alpha$ gives formula (6) as above. The optimal construction can be obtained by minimizing $W_{\text {relax }}(\mathbf{F})$ with respect to $\alpha_{1}$ and $\alpha_{2}$, thus obtaining a system of two equations in two unknowns which can be easily solved numerically to give again the standard Gibbs (isotangent or common tangent) construction

$$
\frac{W^{1}\left(\mathbf{F}_{2}\right)-W^{0}\left(\mathbf{F}_{1}\right)}{\alpha_{2}-\alpha_{1}}=\frac{\partial W^{1}\left(\mathbf{F}_{2}\right)}{\partial \alpha_{2}}=\frac{\partial W^{0}\left(\mathbf{F}_{1}\right)}{\partial \alpha_{1}}
$$

[exploiting the fact that $\partial W^{0}\left(\mathbf{F}_{1}\right) / \partial \alpha_{1}=2 \alpha_{1}$, the system of two equations can easily be reduced to a single equation]. The solution is that lamination is effective when $\alpha$ belongs to the interval

$$
\alpha_{1}^{\min }\left(\lambda_{2}\right) \leqslant \alpha \leqslant \alpha_{2}^{\max }\left(\lambda_{2}\right)
$$

where $\alpha_{1}^{\min }\left(\lambda_{2}\right)$ and $\alpha_{2}^{\max }\left(\lambda_{2}\right)$ denote the optimal values, corresponding to the lower and upper extremes of this interval, respectively [35]. The proposed construction can be further justified and put on a firmer basis by showing that (a) it is the optimal single lamination when keeping $\lambda_{2}$ constant; (b) it is a local minimum in the energy hypersurface; i.e., the gradient of the relaxed energy with respect to the lamination parameters is zero for $\alpha_{1}=\alpha_{1}^{\min }\left(\lambda_{2}\right)$ and $\alpha_{2}=\alpha_{2}^{\max }\left(\lambda_{2}\right)$. The proof (which uses techniques similar to those employed in Appendix A) is quite lengthy and is not reported here. It can be noted in passing that the solution of Eq. (11) is unique [27].

For definiteness, let us call $W_{\text {plastic }}(\mathbf{F})$ the energy relaxed with respect to plastic laminations-i.e., that obtained by minimizing Eq. (10) with respect to $\alpha_{1}$ and $\alpha_{2}$ in the interval where plastic lamination is effective and coinciding with $W^{0}(\mathbf{F})$ and $W^{1}(\mathbf{F})$, respectively, in the appropriate regions of the phase diagram. The region of the phase diagram in which Eq. (12) is satisfied is the region where plastic lamination is effective and is delimited by the two curves

$$
\alpha_{1}^{\min }\left(\lambda_{2}=t / s\right) \leqslant \alpha=\lambda_{3}-\lambda_{1}=s-\frac{1}{t}, \quad Y S(t, s)=0,
$$

and

$$
s-\frac{1}{t}=\lambda_{3}-\lambda_{1}=\alpha \leqslant \alpha_{2}^{\max }\left(\lambda_{2}=t / s\right), \quad U L(t, s)=0,
$$

from below and from above, respectively, furnishing as a lower limit $\alpha_{1}^{\min }\left(\lambda_{2}\right)$, the yield surface in the $t / s$ phase diagram, $Y S(t, s)=0$, and as an upper limit $\alpha_{2}^{\max }\left(\lambda_{2}\right)$, the surface outside which plastic lamination is no longer effective, $U L(t, s)=0$. Note that the relaxed energy and its derivatives are continuous on the critical lines $Y S(t, s)=0$ and $U L(t, s)$ $=0$. It can be observed that, neglecting the slight dependence of $\alpha_{1}^{\min }\left(\lambda_{2}\right)$ on $\lambda_{2}$ (which is accurate for small strain), the curve $Y S(t, s)=0$ reduces to the condition $\alpha=\lambda_{3}-\lambda_{1}=$ cost, which essentially corresponds to the Tresca yield criterion; see Sec. V A. It is also useful to note that the optimal $\alpha_{2}^{\max }$ is 
so large as to completely bypass the "liquid" region of the phase diagram of the $W^{1}(\mathbf{F})$ term. This follows from the fact that the end point of the Gibbs construction must have a positive derivative in the $\lambda_{2}$ direction and thus must lye beyond the minimum of the $W^{1}(\mathbf{F})$ curve.

\section{Neighborhood of the $t=\sqrt{s}$ line}

The approximate construction is not complete yet because it turns out that the plastic lamination is unstable with respect to a second-order lamination in the proximity of the curve $t=\sqrt{s}$. In fact, $W_{\text {plastic }}(\mathbf{F})$ is, by construction, monotonically increasing as a function of $s$ or $t$ along lines $\lambda_{2}=$ const. However, if we keep $\lambda_{3}=s$ constant and we move downwards in the phase diagram starting from the line $\lambda_{2}=1, W_{\text {plastic }}(\mathbf{F})$ is decreasing with decreasing $t$ only in a neighborhood of the line $\lambda_{2}=1$, while passing through a minimum and then starting to increase before getting to the line $t=\sqrt{s}$. It is easy to see, in fact, that the derivative of $W_{\text {plastic }}(\mathbf{F})$ with respect to $t$ keeping $s$ constant is positive for $\lambda_{2}=1$ and negative for $t$ $=1$ by virtue of the constraints in Eq. (12). Let us call $Z D(t, s)=0$ the line of the phase diagram where the derivative of $W_{\text {plastic }}(\mathbf{F})$ with respect to $t$ keeping $s$ constant equals zero. Now, the $\mathbf{F}$ tensors lying on this line can be reached from below through a different lamination, essentially equivalent to that used by DeSimone and Dolzmann in the smectic regions of the phase diagram [31]. Assuming to work for the sake of simplicity in the reference system in which a generic deformation tensor $\mathbf{F}$-lying below the line $Z D(t, s)=0$ - is diagonal, we construct a lamination of $\mathbf{F}$ keeping $\lambda_{3}$ constant as

$$
\begin{aligned}
\mathbf{F}=\left(\begin{array}{ccc}
\lambda_{1} & 0 & 0 \\
0 & \lambda_{2} & 0 \\
0 & 0 & \lambda_{3}
\end{array}\right) & =\frac{1}{2}\left(\begin{array}{ccc}
\lambda_{1} & \omega & 0 \\
0 & \lambda_{2} & 0 \\
0 & 0 & \lambda_{3}
\end{array}\right)+\frac{1}{2}\left(\begin{array}{ccc}
\lambda_{1} & -\omega & 0 \\
0 & \lambda_{2} & 0 \\
0 & 0 & \lambda_{3}
\end{array}\right) \\
& =\frac{1}{2} \mathbf{F}_{1}+\frac{1}{2} \mathbf{F}_{2},
\end{aligned}
$$

where $\omega$ is chosen in such a way that $\mathbf{F}_{1}, \mathbf{F}_{2}=\mathbf{F}^{\text {critical; }}$ i.e., $\mathbf{F}_{1}, \mathbf{F}_{2}$ lie on the line $Z D(t, s)=0$. The corresponding relaxed energy will be

$$
\begin{aligned}
W_{\text {relax }}(\mathbf{F}) & =\frac{1}{2} W_{\text {plastic }}\left(\mathbf{F}_{1}\right)+\frac{1}{2} W_{\text {plastic }}\left(\mathbf{F}_{2}\right) \\
& =W_{\text {plastic }}\left(\mathbf{F}^{\text {critical }}\right)=\left.W_{\text {plastic }}(\mathbf{F})\right|_{Z D\left(t, \lambda_{3}\right)=0}
\end{aligned}
$$

and will therefore be constant as a function of $t$ along the vertical line $\lambda_{3}=s=$ const from the intersection with the line $t=\sqrt{s}$ up to the intersection with the line $Z D(t, s)=0$. Note that the relaxed energy and its derivatives are continuous on the line $Z D(t, s)=0$. Furthermore, they join smoothly with the convex envelope of the plastic $W^{1}(\mathbf{F})$ wells at

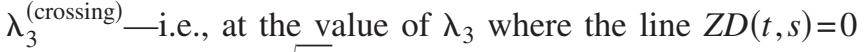
crosses the line $t=\sqrt{\eta s}$, which delimits the upper border of one of the smectic regions of the phase diagram of the $W^{1}(\mathbf{F})$ term. It can be noted in passing that in the case of double laminations the construction is known to be nonunique [27].

The approximate relaxed energy (in the lower half of the phase diagram) is then finally given by the following expression:

$$
W_{\mathrm{r} 2}(\mathbf{F})= \begin{cases}W^{0}(\mathbf{F}) & \text { if } \alpha \leqslant \alpha_{1}^{\min }\left(\lambda_{2}\right), \\ W_{\text {plastic }}(\mathbf{F}) & \text { if } \alpha_{1}^{\min }\left(\lambda_{2}\right) \leqslant \alpha \leqslant \alpha_{2}^{\max }\left(\lambda_{2}\right) \text { and } Z D(t, s) \geqslant 0, \\ W_{\text {critical }}(\mathbf{F}) & \text { if } \lambda_{3} \leqslant \lambda_{3}^{\text {(crossing) }} \text { and } Z D(t, s) \leqslant 0, \\ \delta U+\frac{\lambda_{3}^{2}}{\eta^{2}}+\frac{2 \eta}{\lambda_{3}}-3 & \text { if } \lambda_{3} \geqslant \lambda_{3}^{\text {(crossing) }} \text { and } t \leqslant \sqrt{\eta s}, \\ W^{1}(\mathbf{F}) & \text { if } \alpha \geqslant \alpha_{2}^{\max }\left(\lambda_{2}\right) \text { and } t \geqslant \sqrt{\eta s},\end{cases}
$$

where $W_{\text {critical }}(\mathbf{F})=\left.W_{\text {plastic }}(\mathbf{F})\right|_{Z D\left(t, \lambda_{3}\right)=0}$; see Eq. (15). The corresponding phase diagram is shown in Fig. 3.

\section{B. Convexity: Numerical tests}

We start by observing that Eq. (16) corresponds to a finite-value function. To satisfy the constraint of incompressibility, one needs to extend the definition of $W_{\mathrm{r} 2}(\mathbf{F})$ in Eq. (16) by setting the energy to $+\infty$ when $\operatorname{det}(\mathbf{F}) \neq 1$ :

$$
W^{\mathrm{lc}, \mathrm{i}}(\mathbf{F})= \begin{cases}W_{\mathrm{r} 2}(\mathbf{F}) & \text { if } \operatorname{det}(\mathbf{F})=1, \\ +\infty & \text { if } \operatorname{det}(\mathbf{F}) \neq 1 .\end{cases}
$$

The construction of Eqs. (16) and (17) has many nice features; as it is a natural generalization of the twodimensional case, it is locally stationary and it has a clear appeal to physical intuition. Equation (16) is defined in terms of a finite (maximum two) set of laminates. We will now show that it also corresponds to the lamination (or rank-1) convex envelope of $W_{\text {unrelax }}(\mathbf{F})$ in Eq. (3)-i.e., that further laminations cannot lower the energy. Due to the huge difficulties one runs into when trying to deal with the complicated analytical expressions generated by constructing laminates of Eq. (3), we resorted to numerical tests. These cannot pretend to have the same compelling evidence of rigorous mathematical proofs, but are more widely generalizable and 
applicable to cases (such as the present one) in which analyic proofs are exceedingly difficult.

Two independent approaches were used to prove rank-1 convexity of Eq. (17).

In the first approach, we followed the lines of Refs. $[36,37]$. We thus chose a given $\mathbf{F}$, the deformation tensor, in diagonal form: $\mathbf{F}=\operatorname{diag}\left\{\lambda_{1}, \lambda_{2}, \lambda_{3}\right\}$, with $\lambda_{1} \lambda_{2} \lambda_{3}=1$. We then considered a general rank-1 (single) lamination of $\mathbf{F}$ into $\mathbf{F}_{1}$ and $\mathbf{F}_{2}$ as

$$
\begin{gathered}
\mathbf{F}_{2}=\mathbf{F}\{\mathbf{I}+\nu N|m\rangle\langle n|\}, \\
\mathbf{F}_{1}=\mathbf{F}\{\mathbf{I}-(1-\nu) N|m\rangle\langle n|\},
\end{gathered}
$$

where $\mathbf{I}$ is the identity, $\nu \in[0,1], N \in \mathbf{R}^{+}$, and $|m\rangle$ and $|n\rangle$ are orthonormal vectors. The best possible single lamination corresponds to the minimum of the partially relaxed energy,

$$
W^{\text {relax }}(\mathbf{F})=\nu W^{0}\left(\mathbf{F}_{1}\right)+(1-\nu) W^{1}\left(\mathbf{F}_{2}\right),
$$

as a function of the lamination variables. A general lamination such as this is defined in terms of five degrees of freedom: $\nu, N$, and the three angles defining $|m\rangle$ and $|n\rangle$. Actually, one of these variables-say, $\nu$ - can be obtained analytically by a simple minimization of $W^{\text {relax }}(\mathbf{F})$, thus leaving only four degrees of freedom. For each $\mathbf{F}$ in a properly selected set (see below), we then searched for the best possible lamination in this four-dimensional space through two different possibilities: an equispaced grid containing about 50 points for each degrees of freedom and a random selection of $10^{7}\{N,|m\rangle,|n\rangle\}$ sets (of course, more sophisticated procedures could have been applied, such as global optimization approaches, etc., but we found that these simple search schemes were sufficient in such a low-dimensional space). The phase diagram of Fig. 3 was sampled in the relevant region (i.e., for $\alpha \leqslant 1.3$ ) with an equispaced grid containing 200 points. The parameters of the models were chosen as to be physically reasonable for BPA-PC at RT: $\mu$ $=0.9 \mathrm{GPa}, \eta=1.55$, and $\delta U=0.431$. It turned out that the best possible lamination always coincided with the plastic lamination in the region where this was effective. The lowest branch of the exact lamination tree for $W_{\mathrm{r} 2}(\mathbf{F})$ in Eq. (16) must therefore coincide with a plastic lamination. Having determined the lowest branch of the exact lamination tree, we then considered a double lamination; i.e., for each $\mathbf{F}$ we considered a lamination of the type given in Eq. (18), but in which $\mathbf{F}_{1}$ and $\mathbf{F}_{2}$ were themselves simple laminates if they belonged to the region of the phase diagram where plastic lamination was found to be effective. Again, we searched for the best possible $\mathbf{F}_{1}$ and $\mathbf{F}_{2}$-i.e., those minimizing $W^{\text {relax }}$-in the five-dimensional space defined by $\nu, N,|m\rangle$, and $|n\rangle$ through two different possibilities: an equispaced grid containing about 50 points for each degrees of freedom and a random selection of $10^{7}\{\nu, N,|m\rangle,|n\rangle\}$ sets. We found that double lamination was effective only in the region of the phase diagram in which $Z D(t, s) \leqslant 0$ and that in this region the best possible double lamination coincided with that proposed in Eq. (16). A final search of triple laminations-i.e., laminations of the type given in Eq. (18), but in which $\mathbf{F}_{1}$ and $\mathbf{F}_{2}$ were themselves simple laminates or double lami- nates, if they belonged to the appropriate regions of the phase diagram - turned out to be ineffective. We underline that this is a general approach to construct the lamination convex envelope of a given unrelaxed energy expression. Its constructive character makes it appealing for complicated situations in which physical intuition does not provide much help. The approach is particularly viable from a computational point of view in the present isotropic case, as the phase diagram reduces to three (in the compressible case) or two (in the incompressible case) degrees of freedom, and the CPU and memory storage requirements become trivial by modern computational standards, but it is in principle applicable in general $[36,37]$.

In the second approach [38], we chose a given $\mathbf{F}$, the deformation tensor, in diagonal form: $\mathbf{F}=\operatorname{diag}\left\{\lambda_{1}, \lambda_{2}, \lambda_{3}\right\}$, with $\lambda_{1} \lambda_{2} \lambda_{3}=1$. We then defined two modified deformation tensors $\mathbf{F}_{+}$and $\mathbf{F}_{-}$as

$$
\mathbf{F}_{ \pm}=\mathbf{F}\{\mathbf{I} \pm \delta|m\rangle\langle n|\}
$$

where $\delta>0$ is an infinitesimal quantity and $|m\rangle$ and $|n\rangle$ are orthonormal vectors. The local rank-1 convexity of $W_{\mathrm{r} 2}$, Eq. (16), at $\mathbf{F}$ with respect to the lamination defined by $|m\rangle$ and $|n\rangle$ was checked by numerically evaluating its second derivative with respect to $\delta$ as

$$
\frac{\partial W_{\mathrm{r} 2}(\mathbf{F})}{\partial \delta^{2}}=\frac{W_{\mathrm{r} 2}\left(\mathbf{F}_{+}\right)+W_{\mathrm{r} 2}\left(\mathbf{F}_{+}\right)-2 W_{\mathrm{r} 2}(\mathbf{F})}{\delta^{2}} .
$$

The value of $\delta$ was fixed at $10^{-5}$ : smaller values (down to $10^{-14}$ ) or larger values (up to $10^{-3}-10^{-4}$ ) were checked to produce the same results up to the numerical accuracy, which was set to quadrupole precision to be on the safe side. For each $\mathbf{F}$, (i.e., for each set $\left\{\lambda_{1}, \lambda_{2}, \lambda_{3}\right\}$ ), $10^{7}\{|m\rangle,|n\rangle\}$ pairs were randomly generated. The phase diagram of Fig. 3 was sampled in the relevant region (i.e., for $\alpha \leqslant 1.3$ ) with an equispaced grid containing 200 points. The parameters of the models were chosen as above. In all cases, it was found that the second derivative of $W_{\mathrm{r} 2}$ with respect to $\delta$ was always positive and larger than the numerical accuracy (minimal typical values were around 0.003 ).

The construction of Eq. (16) was thus numerically confirmed to coincide with the lamination envelope of Eq. (3) both directly (constructively) via the first approach and indirectly (by the check of local rank-1 convexity) via the second approach.

It is known that rank-1 convexity does not imply quasiconvexity [22]. The strategy employed in Ref. [31] to prove quasiconvexity passes through the definition of polyconvexity. We recall [26] that the polyconvex (respectively, convex, etc.) envelope $\phi^{\mathrm{pc}}$ of a function $\phi$ is the largest polyconvex function less than or equal to $\phi$, that a function $\phi: \mathbb{R}^{3 \times 3} \rightarrow \mathbb{R}$ is polyconvex if there exists a convex function $h: \mathbb{R}^{19} \rightarrow \mathbb{R}$ such that $\phi(\mathbf{F})=h[\mathbf{F}, \operatorname{cof} \mathbf{F}, \operatorname{det}(\mathbf{F})]$, and that, given finitely many polyconvex functions $\left\{z_{i}\right\}$ and a function $\zeta$ convex and nondecreasing in its arguments, all functions $\phi$ such that $\phi(\mathbf{F})=\zeta\left(\left\{z_{i}\right\}\right)$ are also polyconvex. It is known [26] that $W^{\mathrm{pc}}(\mathbf{F})$ gives a lower bound to the quasiconvex envelope $W^{q c}(\mathbf{F})$ and to the lamination convex envelope $W^{\mathrm{lc}}(\mathbf{F})$. For a finite-value function, polyconvexity in fact implies both qua- 
siconvexity and rank-1 convexity. Even for extended realvalued functions, it follows from a result by Müller and Šveràk [39] that the quasiconvex envelope coincides with the polyconvex and lamination convex envelopes when these coincide and are continuous and finite valued when $\operatorname{det}(\mathbf{F})=1$. In order to prove that the lamination convex envelope $W^{\mathrm{lc}}(\mathbf{F})$ is quasiconvex, it would suffice therefore to show that it is polyconvex. The point is, however, that the variables $s$ and $t$ (which we used to draw the phase diagram of Fig. 3) are polyconvex functions of $\mathbf{F}$, but $W_{\mathrm{r} 2}$ is not a convex function of $s$ and $t$; nor were we able to find other polyconvex functions of $\mathbf{F}$ of which $W_{\mathrm{r} 2}$ is a convex and nondecreasing function. Furthermore, polyconvexity is a strictly nonlocal property, which is extremely difficult to test numerically in three dimensions. The issue of the quasiconvexity of the construction of Eq. (17) is thus left open. We can only suggest that $W_{\mathrm{r} 2}$ being rank-1 convex, it represents the only physically accessible response of the system to deformation.

\section{KINETICS}

BPA-PC exhibits plastic unloading and obeys a ratesensitivity law [40] of the power form $\sigma^{0} \propto \dot{\epsilon}^{0.06}$, with $\dot{\epsilon}$ the strain rate and $\sigma^{0}$ the yield stress. We wish to extend the elastic relaxation results to account for the irreversible behavior of BPA-PC. To this end we consider the effect of kinetics. The ansatz is that microstructures take the form of laminates, as shown in Sec. III A, and dissipation is supported on the interfaces and restricts their motion [41]. The effective behavior can be written as a kinetic law for the motion of the interfaces, and the (attractive) equilibrium configurations are given by the construction of Sec. III A [42]. From a physical point if view, the energy barriers to plastic lamination now assume a fundamental role. From the computations of Ref. [19], in good agreement with experimental data, it turns out that the additional activation energy is of the same order of magnitude as $\delta U$ and cannot thus be neglected, not even in a first approximation. A simple way of introducing kinetic, viscoplastic effects into the model is to use the $\nu$ variables obtained from the lamination construction as internal degrees of freedom and to assume viscous forces connected with $\dot{\nu}$-i.e., with the time-dependent variation of the volume fractions of the various laminates. In the spirit of Refs. $[42,43]$ we must supply a kinetic potential-i.e., a convex function $\psi^{*}(x)$, where $x$ is the derivative of the internal variable with respect to time - and generalize the definition of the free energy by adding this supplementary kinetic term to it. The dynamic problem is so turned into a static one, which can be treated variationally, with obvious advantages from both a fundamental and a numerical point of view. Furthermore, we make the simplifying choice of solving the resulting equations in a time-discretized setting by defining incremental updates of the constitutive equation [42]. In such an approach, the kinetic term to be added to the free energy takes the form

$$
\Delta t \psi^{*}\left(\frac{\Delta \nu}{\Delta t}\right)=\Delta t \psi^{*}\left(\frac{\nu-\nu_{0}}{\Delta t}\right),
$$

where $\nu_{0}$ is the volume fraction of the previous step. To be specific, we take a simple expression for $\psi^{*}(x)$ :

$$
\psi^{*}(x)=K \frac{1}{1+\beta}|x|^{1+\beta},
$$

where the parameter $\beta$ can be derived from the ratesensitivity law $\sigma \propto \dot{\epsilon}^{0.06}$ as $\beta \approx 0.06$. Finally, since the exponent $\beta$ is very small, we approximate this behavior as rate independent by setting $\beta=0$. The expression for the approximate relaxed energy then reads

$$
W_{\text {relax }}(\mathbf{F})=(1-\nu) W^{0}\left(\mathbf{F}_{1}\right)+\nu W^{1}\left(\mathbf{F}_{2}\right)+K\left|\nu-\nu_{0}\right| .
$$

The fact that the additional term $K\left|\nu-\nu_{0}\right|$ is linear in $\left|\nu-\nu_{0}\right|$ corresponds to the hypothesis of rate-independent viscoplasticity [43]. This choice is reasonable for BPA-PC, in which rate-independent viscoplastic effects predominate over rate-dependent ones, and is also particularly convenient, as it allows us to solve Eq. (20) analytically, as will be shown presently.

In our approximate construction we have two different types of lamination: (i) in the neighborhood of the line $\lambda_{2}$ $=1$ (i.e., in shear), one finds a rank-1 plastic lamination between the lower elastic $W^{0}(\mathbf{F})$ well and the upper plastic $W^{1}(\mathbf{F})$ wells; (ii) in the proximity of the lines $t=\sqrt{\mathrm{s}}$ and $t$ $=s^{2}$ (i.e., in tension and compression, respectively), one finds a rank-2 lamination composed of a first balanced lamination, similar to those employed by DeSimone and Dolzmann [see Eq. (15)], combined with a plastic lamination of type (i). Note that in the balanced lamination all the $\nu$ 's assume the constant value $1 / 2$, independently of which variants are being considered, whereas in the plastic lamination the $\nu$ values vary along the phase diagram. Let us analyze the effect of the $K\left|\nu-\nu_{0}\right|$ term on the two types of lamination separately.

Since in the balanced lamination all the $\nu$ 's assume the constant value $1 / 2$, the term $\dot{\nu}$ is formally infinite at the beginning or at the end of a lamination and then identically zero, so that the addition of the term $K\left|\nu-\nu_{0}\right|$ to the unrelaxed energy makes a difference only when creating or destroying an interface. Let us consider for definiteness to load the system starting from a homogenous phase. For a certain value of the strain, we enter the region of the phase diagram where lamination is favorite. However, it will not be realized until the gain in energy becomes greater than $K / 2$ (recall that $\nu_{\mathrm{opt}}$ is $1 / 2, \nu_{0}=0$, before lamination sets in). At this point, a laminate is created, which is exactly the same as the one in the absence of the $K\left|\nu-\nu_{0}\right|$ term [44]. From this point on, the laminate evolves as before, keeping the volume fractions of the variants equal to $1 / 2$ and thus effectively annihilating the $K\left|\nu-\nu_{0}\right|$ term. The effect of the inclusion of this term is thus only to add spikes to the original stress-strain diagram at the beginning and at the end of the lamination. For the rest, the phase diagram of the model with the $K \mid \nu$ $-\nu_{0} \mid$ term included can be trivially obtained from that in the absence of this term.

In the plastic lamination, supposing as always that $\mathbf{F}_{1}$ is evaluated on the elastic well and $\mathbf{F}_{2}$ on one of the plastic wells, we have

$$
W_{\text {relax }}(\mathbf{F})=(1-\nu) W^{0}\left(\mathbf{F}_{1}\right)+\nu W^{1}\left(\mathbf{F}_{2}\right)+K\left|\nu-\nu_{0}\right| .
$$


Working for definiteness in loading conditions, where $\nu>\nu_{0}$, Eq. (21) can be rewritten as

$$
W_{\text {relax }}(\mathbf{F})=(1-\nu) W^{0}\left(\mathbf{F}_{1}\right)+\nu \hat{W}^{1}\left(\mathbf{F}_{2}\right)-K \nu_{0},
$$

where $\hat{W}^{1}\left(\mathbf{F}_{2}\right)=W^{1}\left(\mathbf{F}_{2}\right)+K$; i.e., $\hat{W}^{1}\left(\mathbf{F}_{2}\right)$ is obtained from $W^{1}\left(\mathbf{F}_{2}\right)$ by simply modifying the value of $\delta U$ as $\delta U \rightarrow \delta U$ $+K$. In other words, the phase diagram of the model with the $K\left|\nu-\nu_{0}\right|$ term included is trivially obtained by adding a constant energy shift $\mp K \nu_{0}$ to the energy and by substituting $\delta U$ with $\delta U \pm K$ (with the choice of sign appropriate to loading or unloading conditions). This is tantamount to shifting the onset of the lamination and the value of the yield stress to higher (lower) values if working in loading (unloading) conditions. We thus ended up with the simplest possible model of hysteresis in ideal plasticity. The above is true for the plastic laminations both in the neighborhood of the line $\lambda_{2}$ $=1$ and of the lines $t=\sqrt{s}$ and $t=s^{2}$-i.e., both in shear and in compression or tension. In the latter cases, one also needs to consider the effect of the $K\left|\nu-\nu_{0}\right|$ term for the balanced lamination, which as shown above gives an additional energy barrier to the formation of laminates, thus producing spikes in the stress-strain diagram in tensile or compressive linetests.

It can be recalled that in the case of rank-2 laminations the construction is intrinsically nonunique [27]. However, using the volume fractions $\nu$ 's as internal variables still makes sense under the assumption that, once a rank-2 lamination develops in a given form, it then evolves with continuity.

\section{COMPARISON WITH EXPERIMENT}

In this section, we compare the prediction of the model with experimental results on the mechanical response of BPA-PC in representative cases (behavior of a single volume element), concentrating attention on three topics: (a) yielding criterion and effect of hydrostatic pressure, (b) sample history, and (c) effect of strain rate.

We start with a phenomenological description of the stress-strain diagram of BPA-PC. Extensive mechanical testing have proven that the plastic behavior of BPA-PC proceeds by the nucleation and growth of shear bands (see Ref. [45] and references therein). Local shear strain within the bands is uniform at $\epsilon \approx 0.6$ and remains nearly constant as the bands grow first in length and then in width. A typical experimental stress-strain curve obtained from a shear test on BPA-PC at RT is shown in Fig. 4, taken from Ref. [45]. The deformation typically consists of five distinct stages: (I) An initial homogeneous viscoelastic response at small strains. An upper yield stress $\tau_{0}$ at $\epsilon \approx 0.15$ terminates this stage. (II) A shear stress drop of $\approx 8 \%$ following yield, associated with the formation of a shear band. Material in the band deforms until it attains a shear strain of $\epsilon \approx 0.6$. (III) After strain softening, the stress stabilizes to an almost constant value. The strain within the shear bands is constant, but the band broadens along the axis of the specimen as the nominal shear strain increases. (IV) When the shear band has consumed the gauge length of the specimen, the test piece again strain-

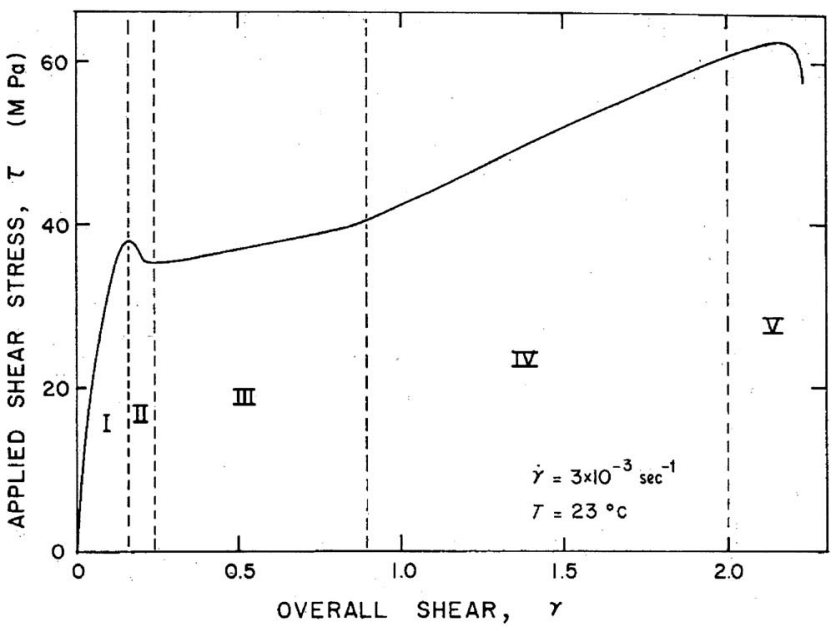

FIG. 4. Typical stress-strain diagram for BPA-PC. Reused with permission from Ref. [45].

hardens homogeneously. (V) Finally, the specimen fractures at a fracture strain $\epsilon \approx 2.0$. It must be stressed that the plastic deformation is fairly uniform within the shear bands, even at a scale as small as $100 \mathrm{~nm}$ [45].

\section{A. Yielding criterion and effect of hydrostatic pressure}

In the literature, the criteria of von Mises or Tresca, properly modified to take into account the dependence of the yield stress upon the hydrostatic pressure [46], have been used to analyze the results of mechanical testing of BPA-PC. The most accurate analysis can be found in Ref. [47]. The experimental results on high-molecular-weight BPA-PC showed a deformation mode intermediate between homogenous and localized yield zones. The modified Tresca criterion most closely reproduced the observed behavior

$$
\tau_{t}=\tau_{0}+\rho \cdot P
$$

where $\tau_{t}$, the maximum shear stress, can be expressed in a terms of the principal stresses $\left\{\sigma_{i}\right\}$,

$$
\tau_{t}=\frac{1}{2}\left(\sigma_{i}-\sigma_{j}\right)_{\max },
$$

and $\tau_{0}$ and $\rho$ are constants. It appears sensible to use a criterion based on the maximum shear stress in the lamellae when the plastic deformation is localized in shear bands as observed in BPA-PC [45]. This is in agreement with our choice that the plastic structures surrounding the elastic one are connected to it via pure shear transformations. We recall that, due to this choice, in our model the yield surface is defined by the equation (see Sec. III A 1)

$$
\left(\lambda_{i}-\lambda_{j}\right)_{\max }=\alpha_{1}^{\min }\left(\lambda_{\text {intermediate }}\right),
$$

which, in the limit of small strain such as those realized for BPA-PC at yielding, closely corresponds to the Tresca criterion. The dependence of the threshold value upon hydrostatic pressure can then be simply accounted for by assuming a dependence of the energy shift $\delta U$ of the plastic structures with respect to the elastic one on the pressure. This 


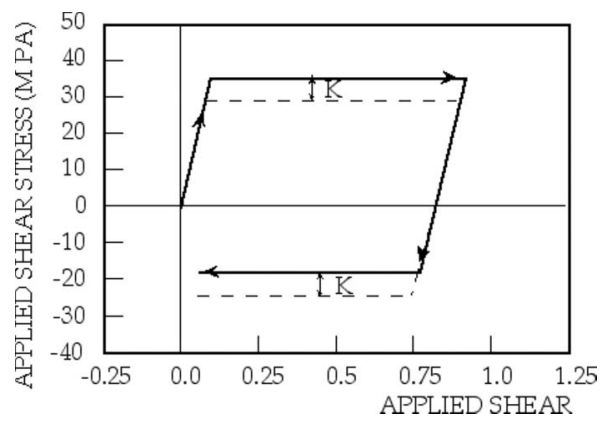

FIG. 5. Stress-strain diagram for a loading-unloading cycle of a representative constitutive element of the proposed construction, Eq. (16), supplemented by a kinetic $K\left|\nu-\nu_{0}\right|$ term. The diagram shows a significant Bauschinger effect. The dotted line represents the response of the system in the absence of the kinetic term. The values of the parameters are $\mu=0.9 \mathrm{GPa}, \eta=1.55, \delta U=0.431$, and $K=0.139$.

assumption is fully reasonable: we recall that in the quadratic approximation (see note [35]) one has $\alpha_{1}^{\min }\left(\lambda_{2}\right)$ $=\delta U \sqrt{\lambda_{2}} /(2 \gamma)$-i.e., a magnification of the energy barriers due to closer packing - as observed also in atomistic simulations [48].

\section{B. Sample history}

In Fig. 5 we show the behavior of a representative element obeying the proposed constitutive equation (16) and subjected first to shear stress and deformation up to the region where laminates are effective and then to a shear stress in the opposite direction. As explained in the previous section, the kinetic term $K\left|\nu-\nu_{0}\right|$ simply increases (decreases) the value of the yield stress to higher (lower) values if working in loading (unloading) conditions by a term $\pm K$. This implies that the yield stress for compression following plastic elongation is smaller than the yield stress of the virgin curve, a phenomenon known as the Bauschinger effect [49] in the plastic yielding of metals. Note that a Bauschinger effect [10] is actually observed for BPA-PC [11] and is also in agreement with the analysis of kinetic effects in Ref. [19].

\section{Effect of strain rate}

It is found experimentally that the yield stress of BPA-PC has a weak power-law dependence on strain rate, quantified by the parameter $\beta[45,50,51]$ :

$$
\beta=\frac{d \sigma_{0}}{d \ln \dot{\epsilon}},
$$

where $\sigma_{0}$ is the yield stress and $\dot{\epsilon}$ is the strain rate. This equation is a phenomenological restating of the Eyring-type [52] equation $\dot{\epsilon}=\dot{\epsilon}_{0} \exp \left[-\left(\Delta G_{0}-v_{0} \sigma_{0}\right) / \mathrm{RT}\right]$, derived from the theory of non-Newtonian viscosity, where $\Delta G_{0}, v_{0}$, and $\dot{\epsilon}_{0}$ are constants. The measured values for the shear activation volume $v_{0}$ are of the order of $3-6 \mathrm{~nm}^{3}$ for BPA-PC $[45,50,51]$. This is much larger than the volume of a monomer unit and confirms that yielding involves the cooperative

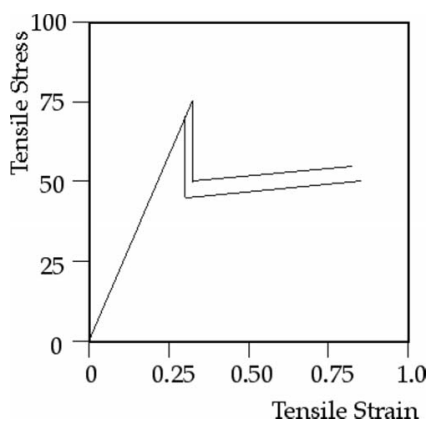

FIG. 6. Stress-strain diagram for a representative constitutive element of the proposed construction, Eq. (16), supplemented by a kinetic $K\left|\nu-\nu_{0}\right|^{1+\beta}$ term (whose effect is taken into account only in the definition of an effective $K$ ) and evaluated at two different strain rates differing by a factor of 20 . The values of the parameters are $\mu=0.9 \mathrm{GPa}, \eta=1.55, \delta U=0.431, K=0.139$, and $\beta=0.065$.

movement of a large number of chain segments. The idea again is that polymer plasticity is controlled by the thermally activated cooperative motion of several chain segments against the elastic constraint of the surrounding matrix. It can be noted that $v_{0}$ decreases to $2 \mathrm{~nm}^{3}$ when $T=-100 \mathrm{C}$ [51], implying a more localized deformation micromechanism at lower temperatures. $\beta$ also depends on temperature, particularly in the range of $T_{g}$, but substantially remains around 0.03-0.06 for BPA-PC in the vicinity of room temperature.

In the previous section, we made the assumption that the additional kinematic term $K\left|\nu-\nu_{0}\right|$ is linear in $\left|\nu-\nu_{0}\right|$, corresponding to the hypothesis of rate-independent viscoplasticity. This allowed us to solve Eq. (20) analytically. In principle, one can introduce a true rate dependence by assuming a form $K\left|\nu-\nu_{0}\right|^{1+\beta}$ for the kinematic term, with $\beta=0.06$. To first order, the effect of the full $K\left|\nu-\nu_{0}\right|^{1+\beta}$ is to change the value of $K$ to an effective value $K \times$ ratio $^{\beta}$, where "ratio" is the ratio of two given strain rates. Figure 6 shows the results of such an approximation for two different values of $\dot{\epsilon}$ (differing by a factor of 20) and a deformation mode corresponding to the pure elongation (tensile test) of a representative constitutive element. This figure compares fairly well with Fig. 7, taken from Ref. [50]. In this connection, an interesting question regards the presence of a stress peak at yield (i.e., a stress drop or strain softening after yield). The origin of this phenomenon is still controversial. It is known that during strain softening the volume decreases by a small amount [46] and the internal energy increases up to a steady value [53] and that the stress peak is strongly dependent upon thermomechanical history of the specimen, external pressure, and temperature [10]. A detailed discussion of this phenomenon is outside the scope of the present work. Here, we simply recall that the stress peak we find from the solution of our model in Fig. 6 is of kinetic origin and is only obtained for deformation close to the lines $\lambda_{2}=\lambda_{1}$ or $\lambda_{3}=\lambda_{2}$-i.e., far from the line $\lambda_{2}=1$ corresponding to a pure shear deformation (for which no stress peak is predicted by our model). In other words, in our model the stress-strain diagram is predicted to exhibit strain softening after yield in tension or compression (due to the rank-2 character of the involved lamination), but not in shear (for which simple 


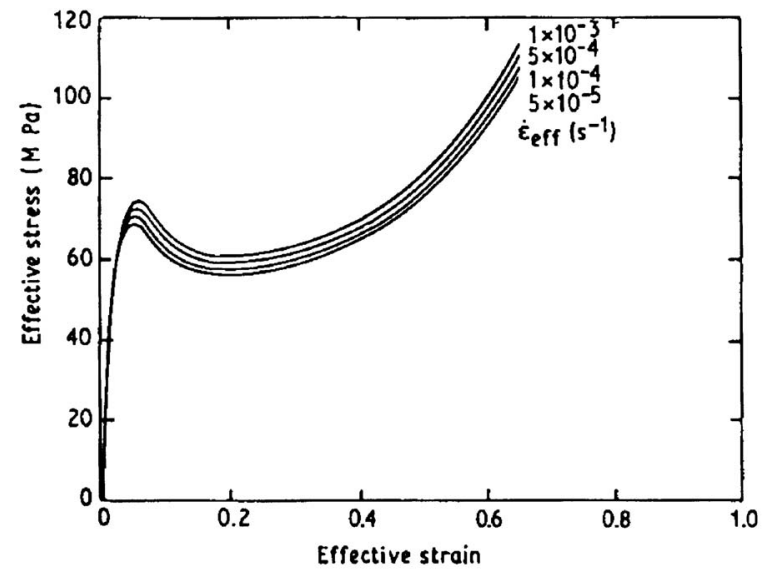

FIG. 7. Dependence of the stress-strain diagram of BPA-PC on the strain rate. Reused with permission from Ref. [50].

plastic laminates are sufficient). It should be taken into account, however, that the plots in Fig. 6 represents the behavior of a single mesoscopic constitutive element. A complete finite-element calculation would be in order to predict the mechanical response to a given mechanical stimulus (elastic misfit stresses, etc., are known to influence the final stressstrain diagram $[54,55]$ ). We finally note that the plastic behavior of amorphous polymers exhibits a noteworthy dependence upon temperature. The inverse relation which is found to exist between yield stress and temperature points to the kinetic nature of yielding as an activated process in the solid state [10]. In our model the effect of temperature can only be accounted for in an indirect way, as it enters into the parameters $\mu, \gamma, \delta U, K$, and $\beta$, which are taken from the results of atomistic simulations or experimental data.

\section{CONCLUSIONS}

A constitutive model for describing the mechanical response of bis-phenol-A-polycarbonate (BPA-PC) has been developed. The model is predicated on recent atomistic simulations of the plastic behavior of BPA-PC [19] and is derived using the tools of the mathematical theory of solidto-solid phase transformations [22,31]. The fundamental observation that serves as the foundation of the model is that plasticity occurs by accommodation of macroscopic deformation through microstructures that exploit the multiwell structure of the elastic energy $[20,22,27]$. Thus, the atomistic simulations reported in [19] reveal that polycarbonate possesses an isotropic elastic phase and an $\mathrm{SO}(3)$ continuum of plastic phases, differing from the parent undeformed structure by a positive energy shift and a finite pure shear transformation. A form of a relaxed energy is proposed, based on a simple lamination construction, and on the strength of extensive numerical tests, it is conjectured that this form represents the lamination-convex envelope of the original freeenergy density. The proposed construction gives rise to ideal elastic-plastic behavior-i.e., to a yield point followed by a zero-slope branch in the stress-strain curve. Kinetics is introduced into the model - and the predicted material behavior is rendered dissipative and hysteretic - by means of a rate- independent interfacial mobility law. This kinetics gives rise to a Bauschinger effect and a double-yield point in tension and compression, but not in shear. These features are in general agreement with the experimental observation of the plastic behavior of BPA-PC.

We conclude by pointing out some of the limitations of the model and suggesting possible extensions. First, different elastic moduli can be accorded to the transformed and untransformed phases at no additional complexity of the model. Experimentally, in fact, it is known that the compliance in the strain-hardening region resembles rubber elasticity rather than the initial elastic regime of amorphous polymers. Second, in order to account for the glassy character of amorphous polymers, a spatial distribution of the parameters $\mu, \gamma$, and $\delta U$ characteristic of the material heterogeneity could be assumed and the effective behavior obtained by homogenization. This extension would effectively smooth out the elastic-plastic transition predicted by the present model, bringing it into closer quantitative agreement with experimental measurements. Third, we note that a broader class of models can be obtained by allowing for more general transformation strains. For instance, following Ref. [31], we may consider the general class of energies

$$
\begin{aligned}
W(\mathbf{F})= & \min \left\{\lambda_{1}^{2}+\lambda_{2}^{2}+\lambda_{3}^{2}-3 ; \delta U+\left(\lambda_{1}^{2} / \chi_{1}\right)^{2}+\left(\lambda_{2}^{2} / \chi_{2}\right)^{2}\right. \\
& \left.+\left(\lambda_{3}^{2} / \chi_{3}\right)^{2}-3\right\},
\end{aligned}
$$

where $\chi_{1} \chi_{2} \chi_{3}=1$ and $\chi_{1} \leqslant \chi_{2} \leqslant \chi_{3}$ represent general transformation stretches. Corresponding approximate rank-1 convex envelopes can then be computed using the approach described in Sec. III. If, by way of example, we chose $\chi_{1}=1$, $\chi_{2}=1, \chi_{3}>1[31]$, the yield surface is approximately given by the condition $\lambda_{3}=$ cost. This model is relevant to nematic elastomers above the transition temperature or to nematic elastomers characterized by polydomain networks [29].

\section{ACKNOWLEDGMENTS}

A.F. wishes to thank Anna Pandolfi (Milano, Italy), Antonio De Simone (Trieste, Italy), and Claudio Geloni and Andrea Lazzeri (Pisa, Italy) for useful discussions, Sergio Conti (Duisburg, Germany) for enlightening discussions, and Matthew Fago (Los Angeles, USA) for numerical tests of the model. He also gratefully acknowledges financial support from Italian CNR in various forms, among which through its Short-Term Mobility Program.

\section{APPENDIX A}

Our goal in this appendix is to express a generic deformation tensor $\mathbf{F}$ with respect to another deformation tensor $\mathbf{F}^{*}$, defined as a pure shear transformation with given norm, and to do this in such a way that the neo-Hookean elastic energy of $\mathbf{F}$ as resulting in the new reference system is minimized. In other words, for any given deformation tensor $\mathbf{F}$ we are looking for the plastic structure closest to it in energy.

Let us consider a generic deformation tensor F. Defining the right Cauchy-Green deformation tensor $\mathbf{C}$ as $\mathbf{C}=\mathbf{F}^{\dagger} \mathbf{F}$, the 
neo-Hookean elastic energy $W^{0}(\mathbf{F})$ is written as the trace of C

$$
W^{0}(\mathbf{F})=\operatorname{tr}(\mathbf{C})-3=\lambda_{1}^{2}+\lambda_{2}^{2}+\lambda_{3}^{2}-3=I_{1}-3,
$$

where $I_{1}$ the first invariant of $\mathbf{C}$ and $\left\{\lambda_{i}^{2}\right\}$ are its eigenvalues, and we have dropped the factor $\mu / 2$ taken as the energy unit.

We now express $\mathbf{F}$ in terms of a different reference deformation tensor $\mathbf{F}^{*}$, connected to the original reference through a pure shear transformation:

$$
\mathbf{F}^{*}=\mathbf{I}+|a\rangle\langle n|,
$$

with the constraints $\langle a \mid n\rangle=0$ and $\langle n \mid n\rangle=1$ (i.e., the vectors $|a\rangle$ and $|n\rangle$ are orthogonal to each other and $|n\rangle$ is a unit vector; see Appendix B).

The elastic energy of $\mathbf{F}$ with respect to this new reference system will be

$$
\begin{aligned}
W^{1}(\mathbf{F}) & =W^{0}\left(\mathbf{F F}^{*-1}\right) \\
& =\operatorname{tr}\left(\mathbf{F}^{* \dagger-1} \mathbf{C F}^{*-1}\right)-3 \\
& =\operatorname{tr}(\mathbf{C})-3+\langle a|\mathbf{C}| a\rangle-2\langle a|\mathbf{C}| n\rangle \\
& =\operatorname{tr}(\mathbf{C})-3+f,
\end{aligned}
$$

where we have worked out the definition of $W^{1}(\mathbf{F})$ in terms of matrix elements of $\mathbf{C}$ and we have defined $f$ as the difference between $W^{0}(\mathbf{F})$ and $W^{1}(\mathbf{F})$

$$
f=W^{1}(\mathbf{F})-W^{0}(\mathbf{F})=\langle a|\mathbf{C}| a\rangle-2\langle a|\mathbf{C}| n\rangle .
$$

Our goal is to minimize $f$ with respect to the vectors $|a\rangle$ and $|n\rangle$, with the only constraint that $N$-the norm of $|a\rangle$-be fixed.

Let us first minimize $f$ with respect to the orientation of $|n\rangle$. From Eq. (A2), this corresponds to finding the maximum of $\langle\mathbf{C} a \mid n\rangle$, with $|a\rangle$ and $\mathbf{C}$ given. In the three-dimensional case, by requiring that the constraint $\langle a \mid n\rangle=0$ be satisfied, this can be achieved by taking $|n\rangle$ as the orthogonal complement of $|\mathbf{C} a\rangle$ with respect to $|a\rangle$ via a standard Schmid orthogonalization:

$$
\left|n_{\mathrm{opt}}\right\rangle \propto|\mathbf{C} a\rangle-\frac{\langle\mathbf{C} a \mid a\rangle}{\langle a \mid a\rangle}|a\rangle,
$$

with the appropriate normalization factor-i.e., the norm of the vector on the right-hand side of Eq. (A3):

$$
\left\|\mathbf{C} a-\frac{\langle\mathbf{C} a \mid a\rangle}{\langle a \mid a\rangle} a\right\|=\sqrt{\left\langle a\left|\mathbf{C}^{2}\right| a\right\rangle-\frac{\langle a|\mathbf{C}| a\rangle^{2}}{\langle a \mid a\rangle}}
$$

(note that the argument of the square root is a positive number by virtue of the Schwartz inequality).

By inserting $\left|n_{\text {opt }}\right\rangle$ in Eq. (A2), it turns out that the minimum value of $f$ with respect to $|n\rangle$ is found by substituting $\langle a|\mathbf{C}| n\rangle$ with the normalization factor 25 , so that now $f$ reads

$$
f=\langle a|\mathbf{C}| a\rangle-2 \sqrt{\left\langle a\left|\mathbf{C}^{2}\right| a\right\rangle-\frac{\langle a|\mathbf{C}| a\rangle^{2}}{\langle a \mid a\rangle}}
$$

or, in terms of $N$ and $|m\rangle$,

$$
f=N^{2}\langle m|\mathbf{C}| m\rangle-2 N \sqrt{\left\langle m\left|\mathbf{C}^{2}\right| m\right\rangle-\langle m|\mathbf{C}| m\rangle^{2}} .
$$

We now choose the Cartesian components $\left\{m_{i}\right\}$ of $|m\rangle$ as our variables:

$$
x=m_{1}^{2}, \quad y=m_{2}^{2}, \quad z=m_{3}^{2},
$$

with the constraints

$$
x, y, z \geqslant 0
$$

and

$$
x+y+z=1 \text {, hence } x=1-y-z,
$$

Let us now suppose to work in the reference system in which $\mathbf{C}$ is diagonal, $\mathbf{C}=\operatorname{diag}\left\{\lambda_{1}^{2}, \lambda_{2}^{2}, \lambda_{3}^{2}\right\}$, and let us introduce the constants

$$
k_{z}=\lambda_{3}^{2}-\lambda_{1}^{2}, \quad k_{y}=\lambda_{2}^{2}-\lambda_{1}^{2},
$$

to rewrite $f$ in a convenient form

$$
\begin{aligned}
f= & N^{2}\left(\lambda_{1}^{2} x+\lambda_{2}^{2} y+\lambda_{3}^{2} z\right) \\
& -2 N \sqrt{k_{z}^{2}\left(z-z^{2}\right)+k_{y}^{2}\left(y-y^{2}\right)-2 k_{z} k_{y} y z}
\end{aligned}
$$

or, equivalently,

$$
\begin{aligned}
\frac{f}{N}-\lambda_{1}^{2} N= & N\left(k_{y} y+k_{z} z\right) \\
& -2 \sqrt{k_{z}^{2} / 4-k_{y} y\left(k_{z}-k_{y}\right)-\left(k_{y} y+k_{z} z-k_{z} / 2\right)^{2}} .
\end{aligned}
$$

We now proceed to the change of variable, $z \rightarrow u=k_{y} y$ $+k_{z} z$, with the new variables $u$ and $y$ ranging in the intervals $0 \leqslant u \leqslant k_{z}$ and $0 \leqslant y \leqslant u / k_{y} . f$ then reads

$$
\frac{f}{N}-\lambda_{1}^{2} N=N u-2 \sqrt{k_{z}^{2} / 4-k_{y} y\left(k_{z}-k_{y}\right)-\left(u-k_{z} / 2\right)^{2}},
$$

which is trivially minimized by $y=0$. Moreover, by imposing that the derivative of $f$ with respect to $u$ equal zero, we find the final solution as

$$
\begin{gathered}
x=m_{1}^{2}=\frac{1}{2}\left(1+\frac{N / 2}{\sqrt{1+N^{2} / 4}}\right), \\
y=m_{2}^{2}=0, \\
z=m_{3}^{2}=\frac{1}{2}\left(1-\frac{N / 2}{\sqrt{1+N^{2} / 4}}\right), \\
f_{\text {min }}=N^{2} \frac{\lambda_{3}^{2}+\lambda_{1}^{2}}{2}-\left(\lambda_{3}^{2}-\lambda_{1}^{2}\right) N \sqrt{1+N^{2} / 4},
\end{gathered}
$$

so that in conclusion the minimum energy $W^{1}(\mathbf{F})_{\min }$ can be rewritten as

$$
\begin{aligned}
W^{1}(\mathbf{F})_{\min }= & \lambda_{1}^{2}\left(1+\frac{N^{2}}{2}+N \sqrt{1+\frac{N^{2}}{4}}\right)+\lambda_{2} \\
& +\lambda_{3}^{2}\left(1+\frac{N^{2}}{2}-N \sqrt{1+\frac{N^{2}}{4}}\right) .
\end{aligned}
$$

Defining $\eta^{2}=1+N^{2} / 2+N \sqrt{1+N^{2} / 4}$, so that $1 / \eta^{2}=1$ $+N^{2} / 2-N \sqrt{1+N^{2} / 4}$, and adding a constant shift $\delta U$, Eq. (A9) coincides with Eq. (3). 
In passing, note that if we release the constraint that the norm of $|a\rangle$ be fixed and minimize $f$ also with respect to $N$, we can find for any given deformation tensor $\mathbf{F}$ the pure shear matrix $\mathbf{F}^{*}$ closest in energy to $\mathbf{F}$. A simple calculation gives

$$
\begin{gathered}
N^{2}=\frac{\lambda_{3}^{2}+\lambda_{1}^{2}}{\lambda_{3} \lambda_{1}}-2, \\
f_{\min }=2 \lambda_{3} \lambda_{1}-\lambda_{3}^{2}-\lambda_{1}^{2}<0 .
\end{gathered}
$$

Note that if $\lambda_{2}=1$ (i.e., $\mathbf{F}$ itself is a pure shear deformation), then $N^{2}=\operatorname{tr}(\mathbf{C})-3$ and $W^{1}(\mathbf{F})=0$; i.e., we recover $\mathbf{F}^{*}$ $=\mathbf{F}$. If instead $\lambda_{2} \neq 1$ but $\lambda_{1} \lambda_{2} \lambda_{3}=1$ (i.e., $\mathbf{F}$ is isochoric), then $W^{1}(\mathbf{F})=\lambda_{2}^{2}+2 / \lambda_{2}-3>0$.

\section{APPENDIX B}

In this appendix we consider some alternative formulas for pure shear transformations. Let us consider a deformation tensor $\mathbf{F}$ of the form

$$
\mathbf{F}=\left(\begin{array}{ccc}
1 & 0 & \alpha \\
0 & 1 & 0 \\
0 & 0 & 1
\end{array}\right),
$$

with $\alpha>0$ for convenience. $\mathbf{F}$ is termed pure shear transformation since it does not contain any volumetric deformation (isochoric) and is limited to a deformation along two principal directions, leaving the third one unmodified. For the right Cauchy-Green deformation tensor $\mathbf{C}$ we have

$$
\mathbf{C}=\mathbf{F}^{\dagger} \mathbf{F}=\left(\begin{array}{ccc}
1 & 0 & \alpha \\
0 & 1 & 0 \\
\alpha & 0 & 1+\alpha^{2}
\end{array}\right),
$$

which can be diagonalized to find

$$
\begin{gathered}
\lambda_{3}^{2}, \lambda_{1}^{2}=1+\frac{\alpha^{2}}{2} \pm \alpha \sqrt{1+\frac{\alpha^{2}}{4}}, \\
\lambda_{3}, \lambda_{1}=\sqrt{1+\frac{\alpha^{2}}{4}} \pm \frac{\alpha}{2} .
\end{gathered}
$$

Note that $\operatorname{det}(\mathbf{F})=\lambda_{1} \lambda_{3}=\operatorname{det}(\mathbf{C})=\lambda_{1}^{2} \lambda_{3}^{2}=1$.

Apart from a multiplication by a rotation matrix (irrelevant for the evaluation of the energy), a one-to-one correspondence thus exists between $\mathbf{F}$ written as in Eq. (B1) and a generic matrix with eigenvalues $\left\{\lambda_{1}=\frac{1}{\lambda_{3}}, \lambda_{2}=1, \lambda_{3} \geqslant 1\right\}$ : to recover the value of $\alpha$, simply put

$$
\alpha=\lambda_{3}-\lambda_{1}=\lambda_{3}-\frac{1}{\lambda_{3}}
$$

Note that a pure shear transformation can also be expressed in a different notation as

$$
\mathbf{F}=\mathbf{I}+|a\rangle\langle n|=\mathbf{I}+a \otimes n,
$$

with the constraints

$$
\begin{aligned}
& \langle a \mid n\rangle=a \cdot n=0, \\
& \langle n \mid n\rangle=n \cdot n=1 ;
\end{aligned}
$$

i.e., the vectors $|a\rangle$ and $|n\rangle$ are orthogonal to each other and $|n\rangle$ is a unit vector. The former notation is the Dirac notation, which will be used hereafter. By bringing out the factor associated with the norm of $|a\rangle, N=\sqrt{\langle a \mid a\rangle}$, one finds

$$
|a\rangle=N|m\rangle
$$

$$
\mathbf{F}=\mathbf{I}+N|m\rangle\langle n|,
$$

which shows the equivalence with Eq. (B1).

Analogously, when $\lambda_{2} \neq 1$ one finds a one-to-one correspondence between a deformation tensor $\mathbf{F}$ written as

$$
\mathbf{F}=\left(\begin{array}{ccc}
\frac{1}{\sqrt{\lambda_{2}}} & 0 & \alpha \\
0 & \lambda_{2} & 0 \\
0 & 0 & \frac{1}{\sqrt{\lambda_{2}}}
\end{array}\right)
$$

whose right Cauchy-Green deformation tensor $\mathbf{C}$ reads

$$
\mathbf{C}=\mathbf{F}^{\dagger} \mathbf{F}=\left(\begin{array}{ccc}
\frac{1}{\lambda_{2}} & 0 & \frac{\alpha}{\sqrt{\lambda_{2}}} \\
0 & \lambda_{2}^{2} & 0 \\
\frac{\alpha}{\sqrt{\lambda_{2}}} & 0 & \frac{1}{\lambda_{2}}+\alpha^{2}
\end{array}\right),
$$

and a generic matrix with eigenvalues $\left\{\lambda_{1}\right.$ $\left.=1 /\left(\lambda_{2} \lambda_{3}\right), \lambda_{2}, \lambda_{3}\right\}$, with

$$
\begin{gathered}
\lambda_{3}^{2}, \lambda_{1}^{2}=\frac{1}{\lambda_{2}}+\frac{\alpha^{2}}{2} \pm \alpha \sqrt{\frac{1}{\lambda_{2}}+\frac{\alpha^{2}}{4}}, \\
\lambda_{3}, \lambda_{1}=\sqrt{\frac{1}{\lambda_{2}}+\frac{\alpha^{2}}{4} \pm \frac{\alpha}{2},}
\end{gathered}
$$

where

$$
\alpha=\lambda_{3}-\lambda_{1}=\lambda_{3}-\frac{1}{\lambda_{2} \lambda_{3}} .
$$


[1] I. M. Ward, The Mechanical Properties of Solid Polymers, 2nd ed. (Wiley, Chichester, 1984).

[2] A. S. Argon, Philos. Mag. 28, 839 (1973).

[3] A. S. Argon, in Materials Science and Technology, edited by H. Mughrabi (VCH, Neinheim, 1993), Vol. 6, pp. 461-508.

[4] M. C. Boyce, D. M. Park, and A. S. Argon, Mech. Mater. 7, 15 (1988).

[5] E. M. Arruda and M. C. Boyce, Int. J. Plast. 9, 697 (1993).

[6] L. Anand and M. C. Gurtin, Int. J. Solids Struct. 40, 1465 (2003).

[7] D. Deng, A. S. Argon, and S. Yip, Philos. Trans. R. Soc. London, Ser. A 329, 549 (1989).

[8] D. Deng, A. S. Argon, and S. Yip, Philos. Trans. R. Soc. London, Ser. A 329, 575 (1989).

[9] D. Deng, A. S. Argon, and S. Yip, Philos. Trans. R. Soc. London, Ser. A 329, 613 (1989).

[10] B. Crist, in The Physics of Glassy Polymers, 2nd ed., edited by R. N. Haward and R. J. Young (Chapman Hall, London, 1997), pp. 155-212.

[11] G. G'Sell, H. ElBari, J. Perez, J. Y. Cavaillè, and G. P. Johari, Mater. Sci. Eng., A 110, 223 (1989).

[12] J. Y. Cavaillè, L. David, and J. Perez, Mater. Sci. Forum 336, 499 (2001)

[13] W. G. Johnston, J. Appl. Phys. 33, 2716 (1962).

[14] Z. H. Stachurski, Prog. Polym. Sci. 22, 407 (1997).

[15] A. P. Sokolov, A. Kisliuk, M. Soltwisch, and D. Quitmann, Phys. Rev. Lett. 69, 1540 (1992).

[16] T. Achibat, A. Boukenter, E. Duval, G. Lorentz, and S. Etienne, J. Chem. Phys. 95, 2949 (1991).

[17] S. R. Elliot, Nature (London) 354, 445 (1991).

[18] A. Mermet, E. Duval, S. Etienne, and C. G'Sell, Polymer 37, 615 (1996).

[19] A. Fortunelli, C. Geloni, and A. Lazzeri, J. Chem. Phys. 121, 4941 (2004).

[20] J. M. Ball and R. D. James, Arch. Ration. Mech. Anal. 100, 13 (1987).

[21] S. Müller, Variational Models for Microstructure and Phase Transitions, Lecture Notes No. 2 (Max Planck Institut für Mathematik in den Naturwissenschaften, Leipzig, 1998).

[22] S. Müller, in Calculus of Variations and Geometric Evolution Problems, edited by F. Bethuel, G. Huisken, S. Müller, K. Steffen, S. Hildebrandt, and M. Struwe (Springer, Berlin, 1999).

[23] R. V. Kohn and G. Strang, Commun. Pure Appl. Math. 39, 113 (1982); 39, 139 (1986); 39, 353 (1986).

[24] S. Aubry, M. Fago, and M. Ortiz, Comput. Methods Appl. Mech. Eng. 192, 2823 (2003).

[25] J. Bonnet and R. D. Wood, Nonlinear Continuum Mechanics for Finite Elements Analysis (Cambridge University Press, Cambridge, England, 1997), Chaps. 5.4-5.6.

[26] J. M. Ball, Arch. Ration. Mech. Anal. 63, 337 (1976/1977).

[27] J. M. Ball and R. D. James, Philos. Trans. R. Soc. London, Ser. A 338, 389 (1992).

[28] K. Bhattacharya, Microstructure of Martensite: Why It Forms and How It Gives Rise to the Shape-Memory Effect (Oxford
University Press, Oxford, 2003).

[29] M. Warner and E. M. Terentjev, Prog. Polym. Sci. 21, 853 (1996).

[30] A. DeSimone, Ferroelectrics 222, 275 (1999).

[31] A. DeSimone and G. Dolzmann, Arch. Ration. Mech. Anal. 161, 181 (2002).

[32] S. Conti, A. DeSimone, and G. Dolzmann, Phys. Rev. E 66, 061710 (2002)

[33] M. Šilhavý, J. Elast. 71, 235 (2003).

[34] To give approximate explicit expressions, if $\delta U \ll \eta-1 \ll 1$, one can expand the resulting equations in power series, obtaining $\alpha_{1}^{\min }=\delta U / 2 \gamma, \alpha_{2}^{\max }=\gamma+\delta U /(2 \gamma)$, within which limits the partially relaxed energy reads $W_{\text {relax }}(\mathbf{F})=(\delta U / \gamma)[\alpha$ $-\delta U /(4 \gamma)]=(\delta U / \gamma)\left[\lambda_{3}-\lambda_{1}-\delta U /(4 \gamma)\right]$, where $\gamma=\eta-1 / \eta$.

[35] To give approximate explicit expressions, if $\delta U \ll \eta-1 \ll 1$, one can expand the resulting equations in power series, obtaining $\alpha_{1}^{\min }\left(\lambda_{2}\right)=\delta U \sqrt{\lambda_{2}} /(2 \gamma), \quad \alpha_{2}^{\max }\left(\lambda_{2}\right)=\gamma / \sqrt{\lambda_{2}}+\delta U \sqrt{\lambda_{2}} /(2 \gamma)$, within which limits the partially relaxed energy reads $W_{\text {relax }}(\mathbf{F})=\lambda_{2}^{2}+2 / \lambda_{2}-3+\left(\delta U \sqrt{\lambda_{2}} / \gamma\right)\left[\alpha-\delta U \sqrt{\lambda_{2}} /(4 \gamma)\right]=\lambda_{2}^{2}+\frac{2}{\lambda_{2}}$ $-3+\left(\delta U \sqrt{\lambda_{2}} / \gamma\right)\left[\lambda_{3}-\lambda_{1}-\delta U \sqrt{\lambda_{2}} /(4 \gamma)\right]$.

[36] G. Dolzmann, SIAM (Soc. Ind. Appl. Math.) J. Numer. Anal. 36, 1621 (1999).

[37] X. Wang and Z. Li, Appl. Math. Comput. 185, 19 (2007).

[38] Suggested by Sergio Conti (private communication).

[39] S. Müller and V. Šveràk, J. Eur. Math. Soc. (JEMS) 1, 393 (1999).

[40] C. G'Sell and J. J. Jonas, J. Mater. Sci. 14, 583 (1979).

[41] R. Abeyaratne, K. Bhattacharya, and J. K. Knowles, in Nonlinear Elasticity: Theory and Application, edited by Y. Fu and R. W. Ogden (Cambridge University Press, Cambridge, England, 2000).

[42] M. Ortiz and L. Stainier, Comput. Methods Appl. Mech. Eng. 171, 419 (1999).

[43] J. C. Simó and T. J. R. Hughes, Computational Inelasticity (Springer, Berlin, 1998), Chap. 10.

[44] This argument can be made rigorous by showing that $\nu=1 / 2$ is the optimal value for this kind of lamination.

[45] C. G'Sell and A. J. Gopez, J. Mater. Sci. 20, 3462 (1985).

[46] W. A. Spitzig and O. Richmond, Polym. Eng. Sci. 19, 1129 (1979).

[47] R. Quinson, J. Perez, M. Rink, and A. Pavan, J. Mater. Sci. 32, 1371 (1997).

[48] A. Fortunelli, C. Geloni, and A. Lazzeri (unpublished).

[49] J. Lubliner, Plasticity Theory (Macmillan, New York, 1990), p. 76.

[50] C. G'Sell, J. M. Hiver, A. Dahoun, and A. Souahi, J. Mater. Sci. 27, 5031 (1992).

[51] N. A. Fleck, W. J. Stronge, and J. H. Liu, Proc. R. Soc. London, Ser. A 429, 459 (1990).

[52] H. Eyring, J. Chem. Phys. 4, 283 (1936).

[53] O. A. Hasan and M. C. Boyce, Polymer 34, 5085 (1993).

[54] V. V. Bulatov and A. S. Argon, Modell. Simul. Mater. Sci. Eng. 2, 203 (1994).

[55] A. S. Argon, V. V. Bulatov, P. H. Mott, and U. W. Suter, J. Rheol. 39, 377 (1995). 